\title{
SOME REMARKS ON COMMUTATIVE ALGEBRAS OF OPERATORS ON BANACH SPACES $\left({ }^{1}\right)$
}

\author{
BY \\ D. A. EDWARDS AND C. T. IONESCU TULCEA
}

Introduction. In this paper a series of propositions are given concerning commutative algebras of operators on a Banach space and more especially commutative algebras of scalar operators. A number of the results are known and due to $W$. G. Bade $[1 ; 2]$ but different proofs are given here. The inspiration for this paper, both in the choice of the subject matter and of method, has largely been derived from $[2 ; 7]$ and $[10]$.

The material presented here is divided into four paragraphs. The first, which is introductory, contains various results on spectral families of measures. The principal propositions are contained in paragraphs 2 and 3. Theorem 1, proved in paragraph 2 , is a generalisation of a theorem due to W. G. Bade [2], and almost all the other results of this same paragraph are more or less consequences of it. In paragraph 3 it is shown that, under certain conditions, an algebra of scalar operators can be identified, in a sense made precise below, with a von Neumann algebra. This fact makes it possible to reduce many results concerning algebras of scalar operators or $\sigma$-complete boolean algebras of projections, in Banach spaces, to corresponding results in Hilbert spaces. Various remarks on spectral families of measures are made in paragraph 4.

1. Spectral families. Let $Z$ be a compact space, $C(Z)$ the set of complexvalued continuous functions defined on $Z, B_{0}(Z)$ the set of complex-valued Baire measurable functions defined on $Z$ and $B(Z)$ the set of complex-valued Borel measurable functions defined on $Z\left({ }^{2}\right)$.

Let $X$ be a Banach space, $X^{\prime}$ the dual of $X$ and $\mathcal{F}=\left(\mu_{x, x^{\prime}}\right)_{x \in X, x^{\prime} \in X^{\prime}}$ a family of Radon measures defined on $Z$. We shall say that $\mathcal{F}$ is semi-spectral if:

(1) $\left(x, x^{\prime}\right) \rightarrow \mu_{x, x^{\prime}}$ is a bilinear mapping;

(2) there is a constant $M(\mathcal{F}) \geqq 1$ which satisfies the inequalities $\left\|\mu_{x, x^{\prime}}\right\|$ $\leqq M(F)\|x\|\left\|x^{\prime}\right\|$ for every $x \in X, x^{\prime} \in X^{\prime}$.

We shall say that a function $f \in B(Z)$ is $\mathcal{F}$-negligible if $\left|\mu_{x, x^{\prime}}\right| *(|f|)=0$ for every $x \in X, x^{\prime} \in X^{\prime}$. A Borel measurable set $A \subset Z$ is $\mathcal{F}$-negligible if the characteristic function of $A, \phi_{A}$, is $\mathcal{F}$-negligible. For every function $f \in B(Z)$ we shall put $N_{\infty}(f, \mathcal{F})=\inf E(f)$ where $E(f)$ is the set of numbers $\alpha>0$ for which

Presented to the Society, January 22, 1959; received by the editors Ociober 13, 1958.

( ${ }^{1}$ This paper was sponsored by the Office of Scientific Research, United States Air Force, under contract SAR AF 49 (638)-153.

${ }^{(2)}$ For the definition of Baire sets and Borel sets see [12]. For other definitions and results of integration theory see [6]. 
the set $\{z|| f(z) \mid>\alpha\}$ is $\mathcal{F}$-negligible. If $f$ is continuous then $N_{\infty}(f, \mathcal{F})$ $=\sup _{z \in S}|f(z)|$ where $S$ is the closure of $\bigcup_{x \in X, x^{\prime} \in X^{\prime}} S\left(\mu_{x, x^{\prime}}\right)$; for a Radon measure $\mu$ on $Z, S(\mu)$ will denote its support.

We shall write $B_{0}^{\infty}(Z)=\left\{f \in B_{0}(Z) \mid N_{\infty}(f, \mathcal{F})<\infty\right\}$ and $B^{\infty}(Z)$ $=\left\{f \in B(Z) \mid N_{\infty}(f, \mathcal{F})<\infty\right\} ; B_{0}^{\infty}(Z)$ and $B^{\infty}(Z)$ are algebras and $f \rightarrow N_{\infty}(f, \mathcal{F})$ is a semi-norm on $B_{0}^{\infty}(Z)$ and also on $B^{\infty}(Z)$. It is easy to see that $B_{0}^{\infty}(Z)$ and $B^{\infty}(Z)$ are complete for the semi-norm $f \rightarrow N_{\infty}(f, \mathcal{F})$ and that, for $f, g, h \in B^{\infty}(Z)$, we have $N_{\infty}(f \bar{f}, \mathcal{F})=N_{\infty}(f, \mathcal{F})^{2}$ and $N_{\infty}(g h, \mathcal{F}) \leqq N_{\infty}(g, \mathcal{F}) N_{\infty}(h, \mathcal{F})$.

For each $f \in B^{\infty}(Z)$ we denote by $U_{\mathcal{F}, f}$ the operator in $\mathscr{L}\left(X, X^{\prime \prime}\right)\left({ }^{3}\right)$ which satisfies the equations $\left\langle U_{\mathcal{F}, f} x, x^{\prime}\right\rangle=\int f d \mu_{x, x^{\prime}}$ for all $x \in X, x^{\prime} \in X^{\prime} ; \int$ means always $\int_{z}$. When there will be no ambiguity we shall write only $U_{f}$ instead of $U_{\mathcal{F}, f}$. In what follows we shall always suppose that all the semi-spectral families considered are such that $U_{f} \in \mathfrak{L}(X, X)$ for $f \in C(Z)$ and $U_{1}=I$. It is easy to see that if $\mathcal{F}$ is a semi-spectral family, $f \in B^{\infty}(Z)$ and $U_{f} \in \mathcal{L}(X, X)$, then $U_{f}$ belongs to the strong closure (= weak closure) of $a(\mathcal{F})=\left\{U_{f} \mid f \in C(Z)\right\}$. A family $\mathcal{F}=\left(\mu_{x, x^{\prime}}\right)_{x \in X, x^{\prime} \in X^{\prime}}$ of Radon measures defined on $Z$ is a spectral family if it is semi-spectral and if:

$$
f \cdot \mu_{x, x^{\prime}}=\mu_{U_{f}, x^{\prime}} \quad \text { for all } f \in C(Z), x \in X, x^{\prime} \in X^{\prime} .
$$

If $\mathcal{F}$ is spectral then $f \rightarrow U_{f}$ is a continuous representation of the algebra $C(Z)$, endowed with the semi-norm $f \rightarrow N_{\infty}(f, \mathcal{F})$ into $\mathcal{L}(X, X)$; it follows that $Q(\mathcal{F})$ is an algebra. Conversely if $\mathcal{F}$ is semi-spectral and $f \rightarrow U_{f}$ is a representation of the algebra $C(Z)$ into $\mathscr{L}(X, X)$, then $\mathcal{F}$ is spectral. Let us also remark that (3) implies $f \cdot \mu_{x, x^{\prime}}=\mu_{x,}{ }^{t} U_{f} x^{\prime}$ for every $f \in C(Z), x \in X, x^{\prime} \in X^{\prime} ;{ }^{t} U_{f}$ denotes the Banach adjoint of $U_{f}$.

Let $A$ be a nonempty bounded subset of $X$ and suppose that $\mathcal{F}$ is spectral. For every $f \in B_{0}(Z)$ define $p(f, A)=\sup _{x \in A,\left\|x^{\prime}\right\| \leq 1}\left|\mu_{x, x^{\prime}}\right| *(|f|)$; let $B B_{0}(Z, A)$ $=\left\{f \in B_{0}(Z) \mid p(f, A)<\infty\right\}$. If $T \in \mathcal{L}(X, X)$ and if $T U_{f} x=U_{f} T x$ for all $f \in C(Z)$ and $x \in A$ then it is easy to see that $p(f, T(A)) \leqq\|T\| p(f, A)$ for every $f \in B_{0}(Z)$ and hence that $B_{0}(Z, A) \subset B_{0}(Z, T(A))$. If $A_{1} \subset A_{2}$ then $B_{0}\left(Z, A_{1}\right)$ $\supset B_{0}\left(Z, A_{2}\right)$.

Proposition 1. (i) $B_{0}(Z, A)$ is a linear space and $B_{0}(Z, A) \supset B_{0}^{\infty}(Z)$; (ii) $f \rightarrow p(f, A)$ is a semi-norm on $B_{0}(Z, A)$; (iii) $B_{0}(Z, A)$ is complete with respect to the semi-norm $f \rightarrow p(f, A)$.

Assertions (i) and (ii) are obvious. To prove (iii) let $\left(f_{n}\right)_{1 \leq n<\infty}$ be a sequence of functions belonging to $B_{0}(Z, A)$ for which $\sum_{n=1}^{\infty} p\left(f_{n+1}-f_{n}, A\right)<\infty$. Then for every $x \in A$ and $\left\|x^{\prime}\right\| \leqq 1$ we have $\sum_{n=1}^{\infty}\left|\mu_{x, x^{\prime}}\right| *\left(\left|f_{n+1}-f_{n}\right|\right)<\infty$. Hence $\lim _{n \rightarrow \infty} f_{n}(z)$ exists for $z \notin N_{A}$ where $N_{A}$ is a Baire set which is $\left|\mu_{x, x^{\prime}}\right|$ negligible for every $x \in A$ and $x^{\prime} \in X^{\prime}$. If we put $f(z)=0$ for $z \in N_{A}$ and $f(z)$ $=\lim _{n \rightarrow \infty} f_{n}(z)$ for $z \notin N_{A}$ then $f \in B_{0}(Z)$, and for $n \geqq 1, x \in A$ and $\left\|x^{\prime}\right\| \leqq 1$ we

(3) If $X, Y$ are Banach spaces we shall denote by $\mathcal{L}(X, Y)$ the space of linear continuous mappings of $X$ into $Y$, endowed with the usual norm. 
have $\left|\mu_{x, x^{\prime}}\right|^{*}\left(\left|f-f_{n}\right|\right) \leqq \sum_{j=n}^{\infty}\left|\mu_{x, x^{\prime}}\right|^{*}\left(\left|f_{j+1}-f_{j}\right|\right) \leqq \sum_{j=n}^{\infty} p\left(f_{j+1}-f_{j}, A\right)$ and hence $p\left(f-f_{n}, A\right) \leqq \sum_{j=n}^{\infty} p\left(f_{j+1}-f_{j}, A\right)$. This implies that $f \in B_{0}(Z, A)$ and that $\lim _{n \rightarrow \infty} p\left(f-f_{n}, A\right)=0$.

For each bounded nonempty subset $A \subset X$ we denote by $B_{0}^{1}(Z, A)$ the closure of $B_{0}^{\infty}(Z)$ in $B_{0}(Z, A)$ and by $L^{1}(Z, A)$ the closure of $C(Z)$ in $B_{0}(Z, A)$. It is obvious that $f g \in B_{0}^{1}(Z, A)$ if $f \in B_{0}^{1}(Z, A)$ and $g \in B_{0}^{\infty}(Z)$ and that $f g \in L^{1}(Z, A)$ if $f \in L^{1}(Z, A)$ and $g \in L^{1}(Z, A) \cap B_{0}^{\infty}(Z)$. Also it is easy to prove that if $f \in L^{1}(Z, A) \cap B_{0}^{\infty}(Z)$ then there is a sequence of functions $\left(f_{n}\right)_{1 \leq n<\infty}$ belonging to $C(Z)$ such that we have $\lim _{n \rightarrow \infty} p\left(f_{n}-f, A\right)=0$ and $\left\|f_{n}\right\|$ $=\sup _{z \in Z}\left|f_{n}(z)\right| \leqq N_{\infty}(f, \mathcal{F})$ for $n=1,2, \cdots$. If $T \in \mathcal{L}(X, X)$ is such that $T U_{f} x=U_{f} T x$ for all $f \in C(Z)$ and $x \in A$ then we have $B_{0}^{1}(Z, A) \subset B_{0}^{1}(Z, T(A))$ and $L^{1}(Z, A) \subset L^{1}(Z, T(A))$. If $A_{1} \subset A_{2}$ then $B_{0}^{1}\left(Z, A_{1}\right) \supset B_{0}^{1}\left(Z, A_{2}\right)$ and $L_{0}^{1}\left(Z, A_{1}\right) \supset L_{0}^{1}\left(Z, A_{2}\right)$. In connection with the spaces $B_{0}(Z, A), B_{0}^{1}(Z, A)$, $L^{1}(Z, A)$ and Proposition 1 see also [15].

Proposition 2. For every bounded nonempty subset $A \subset X$ and $f \in B_{0}^{\infty}(Z)$

$$
(1 / M(F)) p(f, A) \leqq \sup _{x \in A}\left\|U_{f} x\right\| \leqq p(f, A) .
$$

The first inequality, $(1 / M(\mathcal{F})) p(f, A) \leqq \sup _{x \in A}\left\|U_{f} x\right\|$, follows from the relations

$$
\begin{aligned}
p(f, A) & =\sup _{x \in A,\left\|x^{\prime}\right\| \leqq 1}\left(\sup _{\|\phi\| 1}\left|\int f g d \mu_{x, x^{\prime}}\right|\right) \\
& \leqq M(F) \sup _{x \in A,\left\|x^{\prime}\right\| \leqq 1}\left|\left\langle U_{f} x, x^{\prime}\right\rangle\right| \leqq M(F) \sup _{x \in \boldsymbol{A}}\left\|U_{f} x\right\| .
\end{aligned}
$$

The second inequality, $\sup _{x \in A}\left\|U_{f} x\right\| \leqq p(f, A)$, is obvious. Hence the proof is complete.

For every $f \in B_{0}(Z)$ let $D(f)=\left\{x \in X \mid f \in B_{0}^{1}(Z,\{x\})\right\}$; using the inequalities (4) (for $A=\{x\}, x \in X$ ) we can easily prove that there is a mapping $U_{\text {, }}$ of $D(f)$ into $X^{\prime \prime}$ such that $\left\langle U_{f} x, x^{\prime}\right\rangle=\int f d \mu_{x, x^{\prime}}$ for every $x \in D(f)$ and $x^{\prime} \in X^{\prime}$. If $f \in L_{0}^{1}(Z,\{x\})\left(\subset B_{0}^{1}(Z,\{x\})\right)$ then $U_{f} x \in X$. Again using the inequalities (4) and the definition of the spaces $B_{0}^{1}(Z, A)$ we see that:

Proposition 3. For every nonempty bounded subset $A \subset D(f)$ and $f \in B_{0}^{1}(Z, A)$

$$
(1 / M(F)) p(f, A) \leqq \sup _{x \in \boldsymbol{A}}\left\|U_{f} x\right\| \leqq p(f, A) .
$$

For every nonempty bounded subset $A \subset X$ let $X(A)$ be the linear space of all bounded families $y=\left(y_{x}\right)_{x \in A}$ of elements belonging to $X$, endowed with the norm: $\|y\|=\sup _{x \in A}\left\|y_{x}\right\| ; X(A)$ is a Banach space. If $\mathcal{F}=\left(\mu_{x, x^{\prime}}\right)_{x \in X, x^{\prime} \in X^{\prime}}$ is a spectral family of measures we shall denote by $a(F, A)$ the closure of the subset $\left\{\left(U_{f} x\right)_{x \in A} \mid f \in C(Z)\right\} \subset X(A)$.

Proposition 4. For every nonempty bounded subset $A \subset X \quad \mathfrak{Q}(\mathcal{F}, A)$ $=\left\{\left(U_{f} x\right)_{x \in A} \mid f \in L^{1}(Z, A)\right\}$. 
Let $f \in L^{1}(Z, A)$ and let $\left(f_{n}\right)_{1 \leq n<\infty}$ be a sequence of functions belonging to $C(Z)$ such that $\lim _{n \rightarrow \infty} p\left(f-f_{n}, A\right)=0$. Then $\lim _{n \rightarrow \infty}\left(\sup _{x \in A}\left\|U_{f_{n}} x-U_{f} x\right\|\right)=0$ and hence $\left(U_{f} x\right)_{x \in A} \in Q(\mathcal{F}, A)$. Conversely let $y=\left(y_{x}\right)_{x \in A} \in Q(\mathcal{F}, A)$. Then there is a sequence $\left(f_{n}\right)_{1 \S n<\infty}$ of functions belonging to $C(Z)$ such that (in $X(A)) y=\lim _{n \rightarrow \infty} y^{n}$ where for each $n=1,2, \cdots, y^{n}=\left(U_{f_{n}} x\right)_{x \in A}$. From (4) we deduce that $\lim _{n, m \rightarrow \infty} p\left(f_{n}-f_{m}, A\right)=0$ and hence that there is a function $f \in L^{1}(Z, A)$ for which $\lim _{n \rightarrow \infty} p\left(f_{n}-f, A\right)=0$. Using (5) we now deduce that $y=\left(U_{f} x\right)_{x \in A}$.

Let $\mathcal{F}$ be a spectral family of measures defined on $Z$. If $U_{g}=U_{h}$, where $g, h \in B^{\infty}(Z)$, then using (3) we deduce that $g-h$ is $\mathcal{F}$-negligible. This remark implies that we can introduce an involution in $Q(\mathcal{F})$ by writing $U_{f}^{*}=U_{\bar{f}}$. The inequalities (4) show that the mapping $T \rightarrow T^{*}$ of $\propto(\mathcal{F})$ onto $\mathscr{Q}(\mathcal{F})$, just defined, is strongly continuous and it can accordingly be extended to the strong closure, $s(Q(F))$, of $Q(F)$; it is clear that the extension is an involution on $s(Q(F))$.

In what follows we shall of ten be concerned with spectral families $\mathcal{F}$ which have the property:

(E) $U_{f} \in \mathcal{L}(X, X)$ for every $f \in B_{0}^{\infty}(Z)$.

Let $S_{0}(Z)$ be the class of all Baire sets $D \subset Z$. For every $D \in S_{0}(Z)$ define $E_{\mathcal{F}}(D)=U_{\phi_{D}}$; then $E_{\mathscr{F}}$ is a strongly countably additive (s.c.a.) spectral measure on $S_{0}(Z)$ such that $E_{\mathscr{F}}(Z)=I$. Conversely, if $E$ is a s.c.a. spectral measure on $S_{0}(Z)$ such that $E(Z)=I$ and if we put $\mu_{x, x^{\prime}}(f)=\int f(z) d\left\langle E(z) x, x^{\prime}\right\rangle$ for all $f \in C(Z), x \in X, x^{\prime} \in X^{\prime}$, then $\mathcal{F}=\left(\mu_{x, x^{\prime}}\right)_{x \in X, x^{\prime} \in X^{\prime}}$ is a spectral family having property $(\mathrm{E})$ and $E_{\mathfrak{F}}=E$.

If $\mathcal{F}$ is a spectral family having property $(\mathrm{E})$ then it is easy to see that $U_{f} \in \mathfrak{L}(X, X)$ for every $f \in B^{\infty}(Z)$. This can be deduced, for instance, using the following result: $\left(^{*}\right)$ Let $\mathcal{F}=\left(\mu_{x, x^{\prime}}\right)_{x \in X, x^{\prime} \in X^{\prime}}$ be a spectral family of measures on a compact space $Z$ having property (E) and let $x \in X$; then there is a positive Radon measure $\nu_{x}$ on $Z$ such that (for $\left.N \in S_{0}(Z)\right) \nu_{x}(N)=0$ if and only if $\left|\mu_{x, x^{\prime}}\right|(N)=0$ for all $x^{\prime} \in X^{\prime}$. Let us remark that every $U_{f}, f \in B^{\infty}(Z)$, is a scalar operator whose resolution of the identity is a s.c.a. spectral measure on $S_{0}\left(\sigma\left(U_{f}\right)\right)$ [10, pp. 341-342, Lemma 6] and that $U_{g h}=U_{g} U_{h}$ for every $g, h \in B^{\infty}(Z)$.

For a spectral family $\mathcal{F}$ having property $(\mathrm{E})$ we have, for each $f \in B^{\infty}(Z)$, the following inequality which may be established by the method used in the proof of Proposition 2, [7, pp. 177-178]:

$$
N_{\infty}(f, \mathcal{F}) \leqq\left\|U_{f}\right\| \leqq M(\mathcal{F}) N_{\infty}(f, \mathcal{F}) .
$$

Let $\mathcal{F}$ be a spectral family on $Z$, having property $(\mathrm{E})$, and let $\beta_{0}(\mathcal{F})$ $=\left\{U_{f} \mid f \in B_{0}^{\infty}(Z)\right\}$ and $B(F)=\left\{U_{f} \mid f \in B^{\infty}(Z)\right\}$. Using (6) we see that $B_{0}(F)$ and $B(F)$ are uniformly closed and that each is algebraically and topologically isomorphic with an algebra $C(\hat{Z})$ where $\hat{Z}$ is a compact space. It is easy to see that $\beta_{0}(\mathcal{F})$ is the smallest uniformly closed algebra containing $\left\{E_{\mathcal{F}}(A) \mid A \in S_{0}(Z)\right\}$. Using the fact that, if $f \in C(Z), N_{\infty}(f, \mathcal{F})=\sup _{z \in S}|f(z)|$, 
where $S$ is the closure of $\bigcup_{x \in X, x^{\prime} \in X^{\prime}} S\left(\mu_{x, x^{\prime}}\right)$, we see that $Q(F)$ is algebraically and topologically isomorphic with $C(S)$ (this last assertion remains true also for spectral families which do not satisfy condition (E)).

2. Commutative algebras of operators. Let $\mathcal{F}=\left(\mu_{x, x^{\prime}}\right)_{x \in X, x^{\prime} \in X^{\prime}}$ be a spectral family of Radon measures defined on a compact space.

THEOREM 1. Let $A$ be a nonempty bounded subset of $X$ and suppose that $T \in \mathcal{L}(X, X)$ is an operator such that: (i) $T U_{f} x=U_{f} T x$ for all $f \in C(Z)$ and $x \in A$; (ii) $(T x)_{x \in A} \in Q(\mathcal{F}, A)$. Then there is a function $g \in L^{1}(Z, A)$ such that: (j) $\|g\| \leqq M(F)\|T\| ;(\mathrm{jj}) U_{0} x=T x$ for every $x$ belonging to the closed linear space $\mathfrak{T}$ spanned by $\bigcup_{x \in A} \propto(\mathcal{F},\{x\})$.

By Proposition 4 there is an $h \in L^{1}(Z, A)$ such that $U_{h} x=T x$ for every $x \in A$. Let $B=\{z\|h(z) \mid \geqq(1+\lambda) M(F)\| T \|\}$ where $\lambda>0$ (we can suppose $T \neq 0)$. It is obvious that $h \phi_{B} \in B_{0}^{1}(Z, A)$. Choose a sequence $\left(h_{n}\right)_{1 \leq n<\infty}$ of functions belonging to $C(Z)$ such that $\lim _{n \rightarrow \infty} p\left(h_{n}-h, A\right)=0$. By (5) this gives $\lim _{n \rightarrow \infty} U_{h_{n}} x=T x$ for each $x \in A$, and hence (since the condition (ii) implies $\mu_{T x, x^{\prime}}=\mu_{x},{ }^{\prime} T x^{\prime}$ for $x \in A$ and $x^{\prime} \in X^{\prime}$ )

$$
\begin{aligned}
\left\langle U_{\phi_{B}} x,{ }^{t} T x^{\prime}\right\rangle & =\int \phi_{B} d \mu_{x,}{ }^{t} T x^{\prime} \\
& =\int \phi_{B} d \mu_{T x, x^{\prime}} \\
& \int \phi_{B} d \mu_{U_{h_{n}} x, x^{\prime}}=\int h \phi_{B} d \mu_{x, x^{\prime}}=\left\langle U_{h \phi_{B}} x, x^{\prime}\right\rangle ;
\end{aligned}
$$

the above equations imply $\left\|U_{\phi_{B}} x\right\|\|T\| \geqq\left\|U_{h \phi_{B}} x\right\|$ for each $x \in A$. Using again (5) we deduce that $p\left(\phi_{B}, A\right)\|T\| \geqq \sup _{x \in A}\left\|U_{\phi_{B}} x\right\|\|T\| \geqq \sup _{x \in A}\left\|U_{h \phi_{B}} x\right\|$ $\geqq(1 / M(F)) p\left(h \phi_{B}, A\right) \geqq(1+\lambda) p\left(\phi_{B}, A\right)\|T\|$. It follows that $p\left(\phi_{B}, A\right)=0$ and hence that $|h(z)| \leqq M(\mathcal{F})\|T\|$ except when $z \in N_{A}$, where $N_{A}$ is a Baire set such that $\left|\mu_{x, x^{\prime}}\right|\left(N_{A}\right)=0$ for every $x \in A$ and $x^{\prime} \in X^{\prime}$. If we define $g$ as follows: $g(z)=h(z)$ if $z \in N_{A}$ and $g(z)=0$ if $z \in N_{A}$, then $g \in L^{1}(Z, A),\|g\| \leqq M(F)\|T\|$ and obviously $U_{0} x=T x$ for each $x \in A$ (in fact we have $p(g-h, A)=0$ ). Take a uniformly bounded sequence $\left(g_{n}\right)_{1 \leqq n<\infty}$ of functions belonging to $C(Z)$ such that $\lim _{n \rightarrow \infty} p\left(g_{n}-g, A\right)=0$. For each $f \in C(Z)$ we have then: $g \in L^{1}\left(Z, U_{f}(A)\right)$, $\lim _{n \rightarrow \infty} p\left(g_{n}-g, U_{f}(A)\right)=0, g f \in L^{1}(Z, A)$ and $\lim _{n \rightarrow \infty} p\left(g_{n} f-g f, A\right)=0$. We deduce that, when $x \in A, U_{g} U_{f} x=\lim _{n \rightarrow \infty} U_{g_{n}} U_{f} x=\lim _{n \rightarrow \infty} U_{f} U_{g_{n}} x=U_{f} U_{g} x$ $=U_{f} T x=T U_{f} x$. Since $\mathfrak{T}$ is the closure of the set of all finite sums $\sum U_{f_{i}} x_{i}\left(f_{i} \in C(Z), x_{i} \in A\right)$ we deduce $(\mathrm{jj})$ and hence the proof of the theorem is complete.

REMARK. The assertion (jj) can be strengthened. We have $U_{\theta}^{p}=T^{p} x$ for every $p=1,2, \cdots$ and $x \in \mathfrak{T}$. In fact suppose the equations valid for $p-1(p>1)$ and remark that $T(\mathscr{T}) \subset \mathscr{T}$. Then for all $x \in A, x^{\prime} \in X^{\prime}$

$$
\begin{aligned}
\left\langle U_{o^{p}} x, x^{\prime}\right\rangle & =\lim _{n \rightarrow \infty} \int g_{n}^{p-1} g d \mu_{x, x^{\prime}}=\lim _{n \rightarrow \infty} \int g d \mu_{x,{ }^{t} U_{o(n, p-1)} x^{\prime}} \\
& =\lim _{n \rightarrow \infty}\left\langle T x,{ }^{t} U_{g(n, p-1)} x^{\prime}\right\rangle=\lim _{n \rightarrow \infty}\left\langle U_{o(n, p-1)} T x, x^{\prime}\right\rangle=\left\langle T^{p} x, x^{\prime}\right\rangle
\end{aligned}
$$


(we denoted $g_{n}^{p-1}$ by $g(n, p-1)$ ); hence $U_{g^{p}}=T^{p} x$ for $x \in A$ and this implies (as in the proof of Theorem 1) $U_{\theta^{p}} x=T^{p} x$ for every $x \in \mathscr{M}$.

If $\mathscr{D} \subset \mathscr{L}(X, X)$ we denote by $s(D)$ the strong closure of $D$ and by $D_{r}(r>0)$ the set $\{T \in \mathscr{D} \mid\|T\| \leqq r\}$. From Theorem 1 we can deduce the following (see $[14])$ :

Corollary 1. If $B \subset s(Q(\mathcal{F}))$ then $\Re_{1} \subset s\left(Q(\mathcal{F})_{M(\mathcal{F})^{2}}\right)$.

If $T \in B_{1}$ and $A$ is a finite part of $X$ then conditions (i) and (ii) are satisfied. Hence there is a $g \in L^{1}(Z, A)$ such that $\|g\| \leqq M(\mathcal{F})$ and $U_{\theta} x=T x$ for $x \in A$. Now let $\left(g_{n}\right)_{1 \leqq n<\infty}$ be a sequence of functions belonging to $C(Z)$ for which $\lim _{n \rightarrow \infty} p\left(g_{n}-g, A\right)=0$ (we can suppose that $\left\|g_{n}\right\| \leqq M(\mathcal{F})$ for $n=1,2, \cdots$, whence $\left.\left\|U_{g_{n}}\right\| \leqq M(\mathcal{F})^{2}\right)$. Then $\lim _{n \rightarrow \infty}\left\|T x-U_{g_{n}} x\right\|=0$ for every $x \in A$ and this implies that $T \in s\left(\mathbb{Q}(\mathcal{F})_{M(\mathcal{F})^{2}}\right)$.

REMARK. From Corollary 1 it follows that if $Q(\mathcal{F})_{1}$ is strongly closed then $Q(\mathfrak{F})$ is strongly closed.

Corollary 2. Suppose that $\mathfrak{F}$ has property (E) and that there exists a denumerable set $A \subset X$ such that the closed linear space spanned by $\bigcup_{x \in A} a(F,\{x\})$ is $X$. Then $s(Q(F))=\left\{U_{f} \in \mathcal{L}(X, X) \mid f \in B_{0}^{\infty}(Z)\right\}=\left\{U_{f} \in \mathfrak{L}(X, X) \mid f \in B^{\infty}(Z)\right\}$.

We can suppose that $\sum_{x \in A}\|x\|<\infty$. If $T \in s(Q(\mathcal{F}))$ we deduce, using Corollary 2 , that there is a directed family $\left(T_{j}\right)_{j \in I}$ of operators belonging to $\mathfrak{Q}(\mathcal{F})$ which converges uniformly to $T$, on $A$. Then by Theorem 1 there is a $g \in B_{0}^{\infty}(Z)$ such that $T=U_{g}$; hence $s(a(F)) \subset\left\{U_{f} \in \mathcal{L}(X, X) \mid f \in B_{0}^{\infty}(Z)\right\}$. Since $\left\{U_{f} \in \mathcal{L}(X, X) \mid f \in B_{0}^{\infty}(Z)\right\} \subset\left\{U_{f} \in \mathfrak{L}(X, X) \mid f \in B^{\infty}(Z)\right\} \subset s(Q(\mathcal{F}))$ the corollary is completely proved.

Let $X$ be a Banach space. We shall say that an algebra $a \subset \mathfrak{L}(X, X)$ has property $\left(\mathrm{P}_{1}\right)$ if there exists a compact space $Z$ and a spectral family $\mathcal{F}=\left(\mu_{x, x^{\prime}}\right)_{x \in X, x^{\prime} \in X^{\prime}}$ of measures defined on $Z$ such that $a=a(\mathcal{F})$. Evidently an algebra $a \subset \mathfrak{L}(X, X)$ has property $\left(\mathrm{P}_{1}\right)$ if and only if there is a compact space $Z$ and a continuous representation of the algebra $C(Z)$ onto $Q$ (we can show that an algebra $Q$ has property $\left(\mathrm{P}_{1}\right)$ if and only if it is algebraically and topologically isomorphic to an algebra $C(Z)$, where $Z$ is a compact space). An algebra $a \subset \mathcal{L}(X, X)$ has property $\left(\mathrm{P}_{2}\right)$ if there is a compact space $Z$ and a spectral family $\mathcal{F}$ on $Z$ having property $(E)$ and such that $Q=Q(F)$. If $X$ is sequentially weakly complete then every algebra having property $\left(\mathrm{P}_{1}\right)$ clearly has property $\left(\mathrm{P}_{2}\right)$.

If an algebra $Q$ has property $\left(\mathrm{P}_{2}\right)$ and $\mathcal{F}$ is a spectral family such that $Q=Q(F)$, then $F$ has property (E). This follows for instance from:

Proposition 5. Let $\mathcal{F}$ be a spectral family. Then $\mathcal{F}$ has property (E) if and only if $a(\mathcal{F})_{1}$ is relatively weakly compact.

It is obvious that $\mathcal{F}$ has property $(\mathrm{E})$ if $Q(\mathcal{F})_{1}$ is relatively weakly compact. The fact that $Q(\mathcal{F})_{1}$ is relatively weakly compact if $\mathcal{F}$ has property (E) fol- 
lows from Theorem 6 , $\left[11\right.$, p. 160] $\left(f \rightarrow U_{f}\right.$ is weakly compact; we take on $\mathcal{L}(X, X)$ the strong topology and we remark that every bounded closed part is complete). Theorem 3.2, [4, pp. 300-301] can also be used for the same purpose.

If $\mathscr{D} \subset \mathfrak{L}(X, X)$ we denote by $D^{(p)}$ the set of all projections belonging to $D$.

CoRollary. If $a \subset \mathfrak{L}(X, X)$ is a strongly closed algebra having property $\left(\mathrm{P}_{2}\right)$ then $\mathrm{Q}^{(p)}$ is a (bounded) complete boolean algebra.

Let $Z$ be the spectrum of $a$ and $\mathcal{F}$ a spectral family on $Z$ such that $a=Q(F)$. Then $a^{(p)}=\left\{E_{\mathscr{F}}(\omega) \mid \omega \in S_{0}(Z)\right\}$ and hence $a^{(p)}$ is bounded (by $M(\mathcal{F}))$. The completeness of $Q^{(p)}$ follows from the fact that $Q(\mathcal{F})_{M(F)}$ is weakly compact and from Theorem 1, [5, pp. 313-314] (see also [13, pp. 162163]).

THEOREM 2. (i) If a has property $\left(\mathrm{P}_{1}\right)$ then $s(Q)$ has property $\left(\mathrm{P}_{1}\right)$; (ii) if a has property $\left(\mathrm{P}_{2}\right)$ then $s(Q)$ has property $\left(\mathrm{P}_{2}\right)$.

Let us prove first (i). Take a compact space $Z$ and a spectral family of measures on $Z$ such that $Q=Q(F)$. Let us introduce an involution on $Q$ as follows: $U_{f}^{*}=U_{f}$ for each $f \in C(Z)$; let us extend this involution by continuity to $s(\mathrm{Q})$. Let $T \in s(\mathrm{Q})$; then by Corollary 1 of Theorem 1 , there is a uniformly bounded directed family of operators belonging to $Q,\left(U_{f(j)}\right)_{j \in I}$, which converges strongly to $T$. A simple use of the inequalities (4) and of the Cauchy inequality gives, for each $x \in X$ and $j \in I:\left\|U_{f(j)} x\right\|^{2} \leqq p(f(j),\{x\})^{2}$ $\leqq M(F)\|x\| p(f(j) \bar{f}(j), \quad\{x\}) \leqq M(F)^{2}\|x\|\left\|U_{f(j)} U_{(j)}^{*} x\right\|$. We deduce $\|T x\|^{2}$ $\leqq M(\mathcal{F})\|x\|\left\|T T^{*} x\right\|$ for every $x \in X$ and hence $\|T\|^{2} \leqq M(\mathfrak{F})\left\|T T^{*}\right\|$. Since this last inequality implies that $s(Q)$ is isomorphic algebraically and topologically with an algebra $C(\tilde{Z})$ the proof of (i) is complete. The second part of the theorem follows from the first, from Proposition 5 and from Corollary 1 of Theorem 1.

3. Strongly closed algebras of operators. For every s.c.a. spectral measure $E$, defined on a tribe $S_{0}(Z)$, we shall write $R(E)=\left\{E(\omega) \mid \omega \in S_{0}(Z)\right\}$. It is obvious that $R(E)$ is a $\sigma$-complete boolean algebra of projections.

Let $E$ be a s.c.a. spectral measure defined on a tribe $S_{0}(Z)$ and let $\mathcal{F}$ be the corresponding spectral family of measures. Let $Z^{\prime}$ be the spectrum of $\bigotimes_{0}(\mathcal{F})$ and $\mathcal{F}^{\prime}=\left(\mu_{x, x^{\prime}}^{\prime}\right)_{x \in X, x^{\prime} \in X^{\prime}}$ a spectral family on $Z^{\prime}$ such that $\bigotimes_{0}(F)=Q\left(F^{\prime}\right)$; it is easy to see that the closure of $\bigcup_{x \in X, x^{\prime} \in X^{\prime}} S\left(\mu_{x, x^{\prime}}^{\prime}\right)$ is $Z^{\prime}$.

Suppose $R(E)$ complete. Then $Z^{\prime}$ is stonean and every measure $\mu_{x, x^{\prime}}^{\prime}$ is normal. For the sake of completeness we give here a direct proof of these assertions. Let $U \subset Z^{\prime}$ be an open subset and let

$$
P^{\prime}=\sup \left\{P \in \mathbb{R}(E) \mid P \leqq U_{\mathcal{F}^{\prime}, \phi_{U}}\right\} .
$$

There is then an open and closed subset $U^{\prime} \subset Z^{\prime}$ such that $P^{\prime}=U_{\mathcal{F}^{\prime}, \phi_{U^{\prime}}}$. If $U-U^{\prime} \neq \varnothing$ we can find a function $g^{\prime} \in C\left(Z^{\prime}\right), g^{\prime} \neq 0$, whose support is con- 
tained in $U-U^{\prime}$. Let $g \in \mathbb{B}_{0}(\mathfrak{F})$ be such that $U_{\mathfrak{F}, g}=U_{\mathcal{F}^{\prime}, g^{\prime}}$ and let $E^{\prime}=E(\{z \mid g(z) \neq 0\})$. By direct computation we obtain $E^{\prime} P^{\prime}=0$ and if $K$ is the support of $g^{\prime}, E^{\prime} \leqq U_{\mathcal{F}^{\prime}, \phi_{K}} \leqq U_{\mathcal{F}^{\prime}, \phi_{U}}$; hence $P^{\prime}<E^{\prime}+P^{\prime} \leqq U_{\mathcal{F}^{\prime}, \phi_{U}}$. It follows necessarily that $U \subset U^{\prime}$ and $P^{\prime}=U_{\mathcal{F}^{\prime}, \phi_{U}}$. Therefore $U^{\prime}-U$ is $\mathcal{F}^{\prime}$-negligible and since $U^{\prime}-\bar{U}$ is open, $U^{\prime}=\bar{U}$. We deduce that $Z^{\prime}$ is stonean and that every measure $\mu_{x, x^{\prime}}^{\prime}$ is normal.

Theorem 3. Let $E$ be a s.c.a. spectral measure defined on a tribe $S_{0}(Z)$ and let $\mathfrak{F}$ be the corresponding spectral family of measures. Suppose that $R(E)$ is complete. Then there is a Hilbert space $H, a$ von Neumann algebra of operators $B$ on $H$ and an algebraic isomorphism $\phi$ of $\beta_{0}(\mathcal{F})$ onto $B$ such that: (i) $\phi$ is bicontinuous when $\Theta_{0}(\mathcal{F})$ and $B$ are endowed with their uniform topologies; (ii) the restriction of $\phi$ to bounded sets is weakly and strongly bicontinuous; (iii) $\phi(h(T))=h(\phi(T))$ for every $T \in \bigotimes_{0}(\mathcal{F}), h \in S_{0}(\sigma(T))$; (iv) $\phi\left(T^{*}\right)=\phi(T)^{*}$ for every $T \in \Theta_{0}(\mathcal{F})$.

Let $Z^{\prime}$ be the spectrum of $\beta_{0}(\mathscr{F})$ and $\mathscr{F}^{\prime}=\left(\mu_{x, x^{\prime}}^{\prime}\right)_{x \in X, x^{\prime} \in X^{\prime}}$ a spectral family on $Z^{\prime}$ such that $B_{0}(\mathcal{F})=\mathfrak{Q}\left(\mathcal{F}^{\prime}\right)$. Then $Z^{\prime}$ is hyperstonean and every measure $\mu_{x, x^{\prime}}^{\prime}$ is normal. Then there is a Hilbert space $H$, a von Neumann algebra $B$ on $H$ and a *-isometry $f \rightarrow T_{f}$ of $C\left(Z^{\prime}\right)$ onto $B$ (see for instance [8]). If we write $\phi\left(U_{\mathcal{F}^{\prime}, f}\right)=T_{f}$, then $\phi$ is an algebraic isomorphism of $B_{0}(\mathcal{F})$ onto $B$ which has properties (i) and (iv). The weak continuity of $\psi$, the mapping inverse to $\phi$, is a consequence of the fact that every measure $\mu_{x, x^{\prime}}^{\prime}$ is normal and hence that there are complex numbers $c_{1}, c_{2}, c_{3}, c_{4}$ and $a_{1}, a_{2}, a_{3}, a_{4} \in H$ such that $\left\langle U_{\mathcal{F}^{\prime}, f} x, x^{\prime}\right\rangle=\sum_{i=1}^{4} c_{i}\left(T_{f} a_{i} \mid a_{i}\right)$. The weak continuity of $\phi$, on bounded sets, follows from the fact that $\beta_{1}$ is weakly compact. Therefore the first part of (ii) is proved. Now let $\beta$ be the set of linear forms $T \rightarrow\left\langle T x, x^{\prime}\right\rangle$, defined on $B_{0}(\mathcal{F})$, which are positive on the elements $T T^{*}$; it is obvious that the topology on $B_{0}(\mathcal{F})$ defined by the set of semi-norms $\{|\rho| \mid \rho \in \mathcal{\rho}\}$ coincide with the weak topology (see the remark at the end of paragraph 4). Denote by $\tau$ the topology on $\beta_{0}(\mathcal{F})$ defined by the set of semi-norms $\left\{T \rightarrow \rho\left(T T^{*}\right)^{1 / 2} \mid \rho \in \mathcal{P}\right\}$. Since $T$ converges to $T_{0}$ in the topology $\tau$ if and only if $\left(T-T_{0}\right)\left(T-T_{0}\right) *$ converges weakly to zero it follows that the restriction of $\phi$ to bounded sets is bicontinuous when $B_{0}(\mathcal{F})$ is endowed with the topology $\tau$ and $B$ with the strong topology. Therefore, to complete the proof of (ii), it is enough to show that on each bounded subset of $B_{0}(\mathfrak{F})$ the strong topology coincides with $\tau$. It is obvious that $\tau$ is weaker than the strong topology. Conversely suppose that the $U_{\mathcal{F}^{\prime}, f}$ are uniformly bounded and that they converge to $U_{\mathcal{F}^{\prime}, f(0)}$ in the topology $\tau$. Then, for every $x \in X, x^{\prime} \in X^{\prime}$, $\lim \int|f-f(0)|^{2} d\left|\mu_{x, x^{\prime}}^{\prime}\right|=0$ and hence $f$ converges in $\left|\mu_{x, x^{\prime}}^{\prime}\right|$-measure to $f(0)$. Since, for fixed $x \in X,\left\{\mu_{x, x^{\prime}}^{\prime} \mid\left\|x^{\prime}\right\| \leqq 1\right\}$ is relatively weakly compact, we have $\lim \int f d \mu_{x, x^{\prime}}^{\prime}=\int f(0) d \mu_{x, x^{\prime}}^{\prime}$, uniformly with respect to $\left\|x^{\prime}\right\| \leqq 1 ; x \in X$ being arbitrary it follows that $U_{\mathcal{F}^{\prime}, f}$ converges strongly to $U_{\mathcal{F}^{\prime}, \text {, J) }}$. Hence the proof of (ii) is complete. The assertion (iii) is an immediate consequence of (ii). 
REMARKs. (1) The involution in $\beta_{0}(\mathcal{F})$ is introduced by $U_{\mathcal{F}^{\prime}, f}=U_{\mathcal{F}^{\prime}, j}$ or, equivalently, by $U_{\mathcal{F}, f}=U_{\mathcal{F}, \bar{f}}$. If $T \in B_{0}(\mathfrak{F})$ and $E^{T}$ is the resolution of the identity of $T$, defined on $S_{0}(\sigma(T))$, it is easily seen that $T^{*}=\int_{\sigma(T)} \bar{\lambda} d E^{T}(\lambda)$. (2) Every $P \in B^{(p)}$ is a self-adjoint projection and $\phi$ is an order isomorphism of $\mathcal{R}(E)=B_{0}(\mathcal{F})^{(p)}$ onto $\mathbb{B}^{(p)}$. (3) In connection with Theorem 3 see also [3, p. 37, Theorem 9.2]. (4) Some of the arguments involved in the proof of Theorem 3 can be avoided if we remark that (for an equivalent norm) $B_{0}(F)$ is an $\mathrm{AW}^{*}$-algebra and use various known results concerning such algebras (see for instance [18]).

CoRollary 1. Let E be a s.c.a. spectral measure, defined on a tribe $S_{0}(Z)$, and $\mathcal{F}$ the corresponding spectral family. Then the following statements are equivalent: (j) $R(E)$ is complete; $(\mathrm{jj}) \beta_{0}(\mathcal{F})=s\left(\bigotimes_{0}(\mathcal{F})\right) ;(\mathrm{jjj}) R(E)=s(\mathbb{R}(E))$.

If $R(E)$ is a complete boolean algebra, (ii) implies that $B_{0}(F)_{1}$ is strongly complete and hence that $\Theta_{0}(\mathscr{F})_{1}=s\left(\Theta_{0}(\mathfrak{F})_{1}\right)$; therefore we have $B_{0}(\mathscr{F})$ $=s\left(\Theta_{0}(\mathcal{F})\right)$. If $\beta_{0}(\mathcal{F})=s\left(\Theta_{0}(\mathcal{F})\right)$ then $\beta_{0}(\mathcal{F})^{(p)}=R(E)$ is obviously strongly closed. If $R(E)=s(R(E))$ it follows from Theorem 1, [5, pp. 313-314], that $R(E)$ is complete.

Remarks. (1) The results stated in Corollary 1 are due to W. G. Bade $[2$, p. 358, Theorem 4.5]; the proof of the implications $(\mathrm{jj}) \rightarrow(\mathrm{jjj}) \rightarrow(\mathrm{j})$ is essentially the same as the one given in [2]. These assertions are justified by the proposition [2, p. 349]: If $\mathcal{G}$ is a $\sigma$-complete boolean algebra of projections in a Banach space then there exists a compact space $Z$ and a s.c.a. spectral measure defined on $S_{0}(Z)$ such that $\mathcal{G}=\mathbb{R}(E)$. (2) From Corollary 1 follows that if $\mathcal{F}$ is a spectral family on $Z$ having property $\left(\mathrm{P}_{2}\right)$ and such that $B_{0}(\mathcal{F})$ is $\sigma$-finite ( $=$ every orthogonal set of projections belonging to the considered algebra is denumerable) then $\Theta_{0}(\mathcal{F})$ is strongly closed; this result can also be reduced to the corresponding one in Hilbert spaces if we use Theorem 3.

Let $H$ be a Hilbert space and $E^{H}$ a s.c.a. spectral measure, defined on a tribe $S_{0}(Z)$, such that $\Re(E) \subset \mathcal{L}(H, H)$. We shall say that $E^{H}$ is self-adjoint if $E^{H}(\omega)$ is self-adjoint for each $\omega \in S_{0}(Z)$.

Corollary 2. Let E be a s.c.a. spectral measure, defined on a tribe $S_{0}(Z)$. Then: (j) there is a Hilbert space $H$, a s.c.a. self-adjoint spectral measure $E^{H}\left(\mathcal{R}\left(E^{H}\right) \subset \mathcal{L}(H, H)\right)$, defined on $S_{0}(Z)$ and an order isomorphism $\phi$ of $\mathbb{R}(E)$ onto $R\left(E^{H}\right)$ which is uniformly, strongly and weakly bicontinuous; (jj) $\phi$ can be extended (in a unique way) to an algebraic isomorphism, of the strongly closed algebra $Q(E)$ spanned by $R(E)$ onto the strongly closed algebra $Q\left(E^{H}\right)$ spanned by $R\left(E^{H}\right)$, having the properties (i)-(iv) (we replace here $B_{0}(\mathcal{F})$ by $Q(E)$ and B by $Q\left(E^{H}\right)$ ) formulated in Theorem 3 ; (jjj) $R(E)$ is complete if and only if $\mathbb{R}\left(E^{H}\right)$ is.

Let $\mathcal{F}$ be the spectral family corresponding to $E$; obviously $\mathbb{R}(E) \subset s(\mathfrak{Q}(\mathfrak{F}))$. By Theorem 3 there is a Hilbert space $H$, a von Neumann algebra $B \subset \&(H, H)$ 
and an algebraic isomorphism $\phi$ of $s(Q(F))$ onto $\mathbb{B}$, which has the properties (i)-(iv). If we take $E^{H}(\omega)=\phi(E(\omega))$, for $\omega \in S_{0}(Z)$, then $E^{H}$ is a s.c.a. selfadjoint spectral measure on $S_{0}(Z)\left(\mathcal{R}\left(E^{H}\right) \subset \mathcal{L}(H, H)\right)$ and $\phi$ is an order isomorphism of $R(E)$ onto $R\left(E^{H}\right)$ which is uniformly, strongly and weakly bicontinuous. Hence the proof of $(j)$ is complete. The results (jj) (we use for its proof Corollary 1 of Theorem 1 ) and (jjj) are now obvious.

REMARKs. (1) An immediate consequence of Corollary 2 (see the remarks which follow Theorem 3 ) is the proposition: On a $\sigma$-complete boolean algebra of projections the weak and the strong topology coincide. (2) Theorem 4.7, [2, p. 359] can be deduced from Corollary 5.3, [17, p. 38] if we use Theorem 3. (3) Using Theorem 3 we can also prove the following proposition: If $a$ is a strongly closed algebra containing $I, \sigma$-finite, generated (in the strong topology) by a denumerable set and having property $\left(\mathrm{P}_{2}\right)$ then there is an operator $T \in Q$ with real spectrum such that every $U \in Q$ is of the form $h(T)$ where $h \in B_{0}^{\infty}(\sigma(T))$.

Using Theorem 3 and, for instance, Corollary 1, [9, p. 57] we deduce the following:

Corollary 3. Let $a(1) \subset \mathfrak{L}\left(X_{1}, X_{1}\right), a(2) \subset \mathfrak{L}\left(X_{2}, X_{2}\right)$ be two strongly closed algebras having property $\left(\mathrm{P}_{2}\right)$ and $\phi$ an algebraic isomorphism of $a(1)$ onto $Q(2)$ such that $\phi\left(T^{*}\right)=\phi(T)^{*}$. Then $\phi$ is bicontinuous when $Q(1)$ and $Q(2)$ are endowed with their uniform topologies. The restriction of $\phi$ to bounded sets is strongly and weakly bicontinuous.

4. Remarks on spectral families having property (E). Proposition (*) stated at the end of paragraph 1 follows from Theorem 1.4 and Lemma 2.3 [4] or from Theorems 1.3 and 1.4 [4]. Instead of Lemma 2.3 or Theorem 1.3 [4] we can use, for the proof of $\left({ }^{*}\right)$, Theorem $2[11]$.

From Theorem 1.4 [4], taking into account the form of the measure $\nu$ (constructed in the proof of this theorem), we can deduce the following result: $\left({ }^{* *}\right)$ Let $\mathfrak{C}$ be a weakly relatively compact set of Radon measures on a compact space $Z$. Then there is a Radon measure $\nu \geqq 0$ such that: (1) $\nu(f)$ $\leqq \sup _{\mu \in \mathfrak{C}}|\mu|(f)$ for $f \geqq 0 ; \nu(N)=0$ (for $N \in S_{0}(Z)$ ) if and only if $|\mu|(N)=0$ for every $\mu \in \mathfrak{C}$. Using $\left({ }^{* *}\right)$ we can immediately prove the following proposition due to W. G. Bade (Theorem 3.1, [2, pp. 351-353]): Let $Z$ be a compact space and $\mathcal{F}=\left(\mu_{x, x^{\prime}}\right)_{x \in X, x^{\prime} \in X^{\prime}}$ a spectral family of measures given on $Z$, having property (E). Then for every $y \in X$ there is a $\nu_{y}=\mu_{y, y^{\prime}} \geqq 0$ such that every $\mu_{y, x^{\prime}}$ is absolutely continuous with respect to $\nu_{y}$. In fact take $\mathbb{C}=\left\{\mu_{y, x^{\prime}} \mid\left\|x^{\prime}\right\| \leqq 1\right\}$ and $\nu_{y}=\nu$. We then have $\nu_{y}(|f|) \leqq p(f,\{y\})$ for every $f \in C(Z)$. Define $\beta$ on $X_{y}=\left\{U_{f} y \mid f \in C(Z)\right\}$ by the equation: $\beta\left(U_{f} y\right)=\int f d \nu_{y}$. Using the inequalities $\nu_{y}(|f|) \leqq p(f,\{y\}) \leqq M(F)\left\|U_{f} y\right\|$ we deduce that $\beta$ is defined on $X_{y}$ without ambiguity and that it is continuous; $\beta$ can therefore be extended to $X$. If we denote the extension by $y^{\prime}$, then $\nu_{y}=\mu_{y, y^{\prime}}$. 
By the same method, but without using proposition $\left({ }^{* *}\right)$ or $\left({ }^{*}\right)$, we prove that every measure $\left|\mu_{x, x^{\prime}}\right|$ is a measure $\mu_{y, y^{\prime}}$.

Let $a$ be a strongly closed algebra having property $\left(\mathrm{P}_{2}\right)$ and $\mathcal{F}$ a spectral family of measures defined on the spectrum of $a$ such that $a=Q(F)$. We have then the following proposition (which we shall state without proof): Every measure $\mu_{x, x^{\prime}}$ is normal and every normal measure on $Z$ is absolutely continuous with respect to a measure $\mu_{x, x^{\prime}} \geqq 0$. In the case of Hilbert spaces more precise results are valid (see [8] and [16]).

\section{REFERENCES}

1. W. G. Bade, Weak and strong limits of spectral operators, Pacific J. Math. vol. 4 (1954) pp. $393-414$.

2. - On boolean algebras of projections and algebras of operators, Trans. Amer. Math. Soc. vol. 80 (1955) pp. 345-360.

3. - A multiplicity theory for boolean algebras of projections in Banach spaces, University of California, Technical Report no. 18, Office of Naval Research, December, 1957.

4. R. J. Bartle, N. Dunford and J. Schwartz, Weak compactness and vector measures, Canad. J. Math. vol. 7 (1955) pp. 289-305.

5. J. Y. Barry, On the convergence of ordered sets of projections, Proc. Amer. Math. Soc. vol. 5 (1954) pp. 313-314.

6. N. Bourbaki, Intégration, Livre VI, Chapters I-V, Paris, 1952-1957.

7. J. Dieudonné, Sur la théorie spectrale, J. Math. Pures Appl. vol. 35 (1956) pp. 175-187.

8. J. Dixmier, Sur certains espaces considérés par M. H. Stone, Summa Brasil. Math. vol. 2 (1951) pp. 151-182.

9. —_ Les algèbres d'opérateurs dans l'espace hilbertien, Paris, 1957.

10. N. Dunford, Spectral operators, Pacific J. Math. vol. 4 (1954) pp. 321-354.

11. A. Grothendieck, Sur les applications linéaires faiblement compactes d'espaces du type $C(K)$, Canad. J. Math. vol. 5 (1953) pp. 129-173.

12. P. Halmos, Measure theory, New York, 1950.

13. E. Hille and R. S. Phillips, Functional analysis and semi-groups, Amer. Math. Soc. Colloquium Publications, vol. 31, 1957.

14. I. Kaplansky, A theorem on rings of operators, Pacific J. Math. vol. 1 (1951) pp. 227-232.

15. W. A. J. Luxemburg, Banach funciion spaces, Delft, Netherlands, 1955.

16. Robert Pallu de la Barrière, Sur les algèbres d'opérateurs dans les espaces hilbertiens, Bull. Soc. Math. France vol. 82 (1954) pp. 1-52. 1951.

17. I. E. Segal, Decompositions of operator algebras II, Memoirs Amer. Math. Soc., no. 9,

18. Ti Yen, Trace on finite AW*-algebras, Duke Math. J. vol. 22 (1955) pp. 207-222.

YALE UNIVERSITy, New Haven. Connecticut 